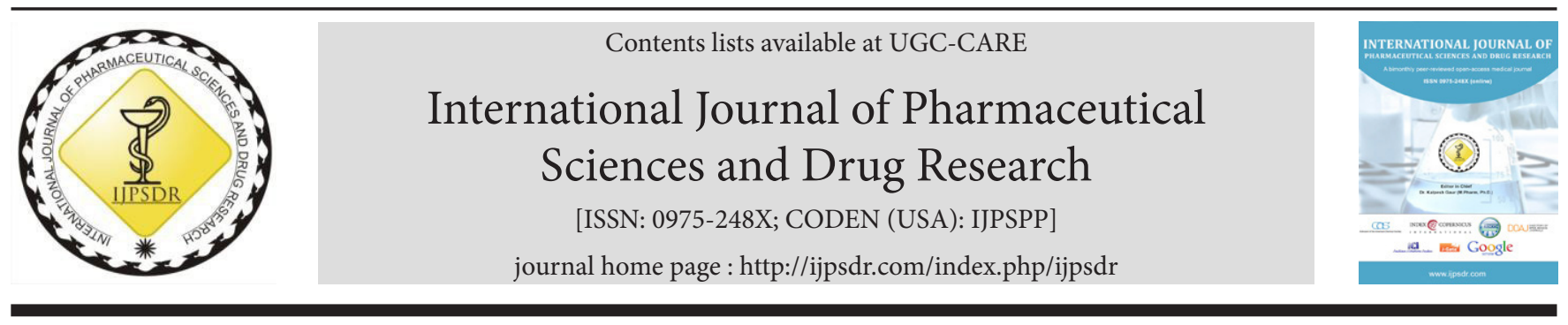

Research Article

\title{
Enhancement of Solubility and Dissolution Profile of Clopidogrel by various Solid Dispersion Formulations
}

\author{
Mohammed Abdul Khader, Roshan Salfi ${ }^{*}$ \\ Department of Pharmacy, Mewar University, Chittorgarh-312901, Rajasthan, India
}

\begin{tabular}{l} 
A R T I C L E I N F O \\
\hline Article history: \\
Received: 24 April, 2020 \\
Revised: 11 June, 2020 \\
Accepted: 26 June, 2020 \\
Published: 30 July, 2020 \\
Keywords: \\
Clopidogrel, \\
Fusion (melt) method, \\
Platelet aggregation inhibitor, \\
Pluronic F127, \\
Solid dispersion. \\
DoI: \\
10.25004/IJPSDR.2020.120410
\end{tabular}

\section{INTRODUCTION}

Drug delivery by oral routes is the fundamental and simpler route of drug administration owing to smaller bulk, precise dosage, and manufacturing ease. ${ }^{[1]}$ Solid dosage forms are advantageous over other oral dosage forms that produce efficient and reproducible in vivo drug plasma concentration post-administration; hence, the majority of new chemical entities (NCE) adopt solid dosage techniques that include tablets, capsules, powders, etc.

As more than $40 \%$ of NCE suffer from poor drug solubility and bioavailability, enhancement of drug solubility has always been a challenge for researchers. Drug solubility and membrane permeability are major factors that limit the drug absorption from gastro intestinal (GI) track. The first step in oral drug delivery is dissolution of drugs in intestinal fluids, which then reaches the bloodstream for circulation. Hence, drugs with poor aqueous solubility exhibit incomplete absorption and permeability through GI track, thus, reducing the drug efficiency. ${ }^{[2,3]}$

Hence, the two major challenges of pharmaceutical drug development include enhancement of solubility of sparingly water-soluble drugs and enhancing penetrations of poorly permeable drugs. ${ }^{[4]} \mathrm{SD}$ technique proved to be an effective tool for meeting these challenges. SDs are a bunch of solid-state elements comprising of two distinct components, usually hydrophilic matrix and a hydrophobic drug.

CPG is a potent oral antiplatelet agent acting by inhibiting platelet activation and aggregation often used in the treatment of coronary artery disease, peripheral vascular disease, and cerebrovascular disease. CPG is

\footnotetext{
"Corresponding Author: Roshan Salfi

Address: Department of Pharmacy, Mewar University, Chittorgarh-312901, Rajasthan, India

Email $₫$ : roshansalfi@yahoo.com

Tel.: +91-8686377725

Relevant conflicts of interest/financial disclosures: The authors declare that the research was conducted in the absence of any commercial or financial relationships that could be construed as a potential conflict of interest.

Copyright (C) 2020 Mohammed Abdul Khader et al. This is an open access article distributed under the terms of the Creative Commons AttributionNonCommercial-ShareAlike 4.0 International License which allows others to remix, tweak, and build upon the work non-commercially, as long as the author is credited and the new creations are licensed under the identical terms.
} 
classified as BCS class II, owing to poor water solubility and high permeability that leads to low oral bioavailability. Hence, it is essential to augment the aqueous solubility and dissolution rate of CPG to obtain faster onset of action, minimize the variability in absorption, and improve their overall oral bioavailability. ${ }^{[5,6]}$

The present study deals with SD formulation of CPG using various carriers, like pluronic F127, labrafac PG, PEG 6000, and gelucire 50/13 for enhancing dissolution and/or solubility and thereby bioavailability of the drug.

\section{Materials AND METHODS}

\section{Materials}

CPG gifted by Hetero Labs Limited, Hyderabad. Poloxamer 407, labrafil PG, PEG 6000, gelucire 50/13, pluronic F127, ethanol, and dichloromethane were obtained from Gattefosse, Mumbai. All the reagents used were of analytical grade.

\section{Methods}

\section{Preliminary Solubility Study of CPG}

Solubility study of CPG carried out by dissolving excess drug in $25 \mathrm{~mL}$ of water-soluble carrier solutions (in distilled water and phosphate buffer of $\mathrm{pH}$ 6.8) in varying ratios in screw-capped bottles. The contents mixed continuously for about 24 hours at room temperatures. The resultant suspension filtered, filtrate analyzed for the $\mathrm{CPG}$ at $240 \mathrm{~nm}$, using UV method. ${ }^{[7]}$

\section{Preparation of CPG SD}

Solvent evaporation method: Twenty formulations (CPG1CPG20) of CPG SD prepared with carriers (pluronic F127, labrafac PG, PEG 6000, and gelucire 50/13) in varying weight ratios by the solvent evaporation method.

Weighed quantity of drug and carriers in dissolved in ethyl alcohol in RB flask and evaporated at $45^{\circ} \mathrm{C}$. The obtained SDs stored in the oven under vacuum at room

Table 1: Preparation of CPG SD by solvent evaporation method (CPG1-CPG10)

\begin{tabular}{llll}
\hline & \multicolumn{2}{l}{ Ingredients quantity $(\mathrm{mg})$} & \\
\cline { 2 - 3 } Solid dispersion code & CPG & Pluronic F127 & Ratio \\
\hline CPG1 & 75 & - & $1: 0.5$ \\
CPG2 & 75 & - & $1: 1$ \\
CPG3 & 75 & - & $1: 2$ \\
CPG4 & 75 & - & $1: 3$ \\
CPG5 & 75 & - & $1: 4$ \\
CPG6 & 5 & - & $1: 0.5$ \\
CPG7 & 75 & - & $1: 1$ \\
CPG8 & 75 & - & $1: 2$ \\
CPG9 & 75 & - & $1: 3$ \\
CPG10 & 75 & - & $1: 4$ \\
\hline
\end{tabular}

temperature for 48 hours for complete evaporation of solvent. The dried SDs grinded using mortar with pestle, sieved through \#60, and stored in desiccator (Tables 1 and 2). ${ }^{[8]}$

Fusion (melt) method: Twenty formulations (CPG21CPG40) of CPG SD prepared using carriers (pluronic F127, labrafac PG, PEG 6000, and gelucire 50/13) in varying ratios by fusion (melt) method.

Weighed quantities of carriers placed on china dish, kept on hot plate and allowed to melt with constant stirring at 50 to $60^{\circ} \mathrm{C}$. Known amount of CPG incorporated into the carrier(s) by constant stirring to attain homogeneity and the mixture heated to obtain clear homogeneous melt. The China dish removed from the hot plate, the melt was transferred onto an aluminum pan and cooled to room temperature. The dried SD was pulverized and sieved through sieve number 60 . The samples were stored in amber-colored bottles capped with rubber corks and kept in desiccators (Tables 3 and 4). ${ }^{[9]}$

\section{Pre-Compression Evaluation Parameters}

The angle of repose, Carr's compressibility index, bulk density, tapped density, and Hausner ratio was performed on the basis of the reported method. ${ }^{[10]}$

\section{Evaluation of CPG SDS}

The solubility study of CPG SD performed as per published method by Higuchi and Connors in $1965 .{ }^{[11]}$ The percentage practical yield, ${ }^{[12]} \%$ drug content, ${ }^{[13]}$ and in vitro drug dissolution study ${ }^{[14]}$ were evaluated as per the referred methods. The SD is further characterized for FTIR analysis, ${ }^{[15]}$ X-ray diffractometer (XRD), ${ }^{[16,17]}$ and SEM studies $^{[18]}$ for drug compatibility studies.

\section{In vitro Drug Dissolution of CPG SD}

The dissolution of CPG from SDs prepared was investigated in $900 \mathrm{~mL}$ phosphate buffer ( $\mathrm{pH}$ 6.8) using USP type II (paddle type) dissolution test apparatus at $50 \mathrm{rpm}$. A temperature of $37 \pm 5^{\circ} \mathrm{C}$ maintained during the course

Table 2: Preparation of CPG SD by solvent evaporation method (CPG11-CPG20)

\begin{tabular}{|c|c|c|c|c|}
\hline \multirow{2}{*}{$\begin{array}{l}\text { Solid dispersion } \\
\text { code }\end{array}$} & \multicolumn{3}{|c|}{ Ingredients quantity (mg) } & \multirow[b]{2}{*}{ Ratio } \\
\hline & $C P G$ & Labrafac $P G$ & Pluronic F127 & \\
\hline CPG11 & 75 & 35 & - & $1: 0.5$ \\
\hline CPG12 & 75 & 75 & - & $1: 1$ \\
\hline CPG13 & 75 & 150 & - & $1: 2$ \\
\hline CPG14 & 75 & 225 & - & $1: 3$ \\
\hline CPG15 & 75 & 300 & - & $1: 4$ \\
\hline CPG16 & 75 & - & 35 & $1: 0.5$ \\
\hline CPG17 & 75 & - & 75 & $1: 1$ \\
\hline CPG18 & 75 & - & 150 & $1: 2$ \\
\hline CPG19 & 75 & - & 225 & $1: 3$ \\
\hline CPG20 & 75 & - & 300 & $1: 4$ \\
\hline
\end{tabular}


Table 3: Preparation of CPG SD by fusion (melt) method (CPG21-CPG30)

\begin{tabular}{llllll}
\hline & \multicolumn{2}{l}{ Ingredients quantity $(\mathrm{mg})$} & & \\
\cline { 2 - 5 } Solid dispersion code & CPG & Gelucire-50/13 & Labrafac PG & Pluronic F127 & - \\
CPG21 & 75 & - & - & - & $1: 0.5$ \\
CPG22 & 75 & - & - & - & $1: 1$ \\
CPG23 & 75 & - & - & - & $1: 2$ \\
CPG24 & 75 & - & - & - & $1: 3$ \\
CPG25 & 75 & - & - & - & $1: 4$ \\
CPG26 & 75 & 35 & - & - & $1: 1$ \\
CPG27 & 75 & 75 & - & - & $1: 2$ \\
CPG28 & 75 & 150 & - & - & $1: 3$ \\
CPG29 & 75 & 225 & - & - & $1: 4$ \\
CPG30 & 75 & 300 & - & \\
\hline
\end{tabular}

Table 4: Preparation of CPG SD by fusion (melt) method (CPG31-CPG40)

\begin{tabular}{|c|c|c|c|c|c|c|}
\hline \multirow[b]{2}{*}{ Solid dispersion code } & \multicolumn{5}{|c|}{ Ingredients quantity $(\mathrm{mg})$} & \multirow[b]{2}{*}{ Ratio } \\
\hline & $C P G$ & PEG 6000 & Gelucire-50/13 & Labrafac $P G$ & Pluronic F127 & \\
\hline CPG31 & 75 & & - & 35 & - & $1: 0.5$ \\
\hline CPG32 & 75 & & - & 75 & - & $1: 1$ \\
\hline CPG33 & 75 & & - & 150 & - & $1: 2$ \\
\hline CPG34 & 75 & & - & 225 & - & $1: 3$ \\
\hline CPG35 & 75 & & - & 300 & - & $1: 4$ \\
\hline CPG36 & 75 & & - & - & 35 & $1: 0.5$ \\
\hline CPG37 & 75 & & - & - & 75 & $1: 1$ \\
\hline CPG38 & 75 & & - & - & 150 & $1: 2$ \\
\hline CPG39 & 75 & & - & - & 225 & $1: 3$ \\
\hline CPG40 & 75 & & - & - & 300 & $1: 4$ \\
\hline
\end{tabular}

of study. The SDs containing $75 \mathrm{mg}$ of CPG dissolved in $5 \mathrm{~mL}$ dissolution media, followed by filtration through a filter $(0.45 \mu)$ at varying time intervals suitably diluted and assayed at $240 \mathrm{~nm}$, and the drug release was compared with the marketed formulation. ${ }^{[19,20]}$

\section{Stability Study}

The CPG SD was sealed in 40 cc HDPE containers under controlled conditions in the stability chamber (Thermo Lab, India) as per ICH guidelines. Samples analyzed for 1,2 , and 3 months for $\%$ drug content and drug release rates. ${ }^{[21]}$

\section{RESULTS}

\section{Pre-Compression Evaluation Parameters}

The angle of repose of all CPG SDs ranged between 21.13 and 26.45. The bulk density of all CPG SDs ranged from 0.5 to $0.59 \mathrm{~g} / \mathrm{cc}$. The tapped density of CPG1 to CPG40 was within the range of 0.61 to $0.69 \mathrm{~g} / \mathrm{cc}$. The compressibility index of CPG1 to CPG40 ranged from 9 to $14 \%$. The Hausner's ratio values of CPG1 to CPG40 in the range of 1.1 to $1.18 \%$. These findings designated that all the formulations had good flow properties.

\section{CPG Solubility Studies}

The solubility study results indicate that the drug solubility is higher in phosphate buffer is $0.146 \mathrm{mg} / \mathrm{mL}$ compared to water $(0.118 \mathrm{mg} / \mathrm{mL})$. The solubility of CPG was highest in polymer pluronic F127 in phosphate buffer pH 6.8 (3.79 mg/mL), which was almost 26 folds compared to drug solubility in $6.8 \mathrm{pH}$ phosphate buffer (Fig. 1).

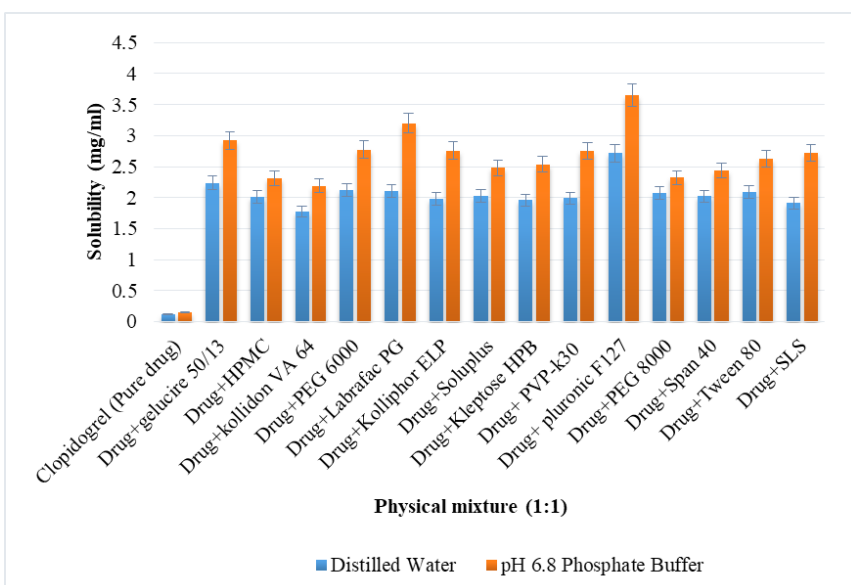

Fig. 1: Solubility studies of CPG in various polymers ${ }^{*}( \pm \mathrm{SD}) \mathrm{n}=3$ 


\section{Solubility Studies of CPG SD}

The results indicate that the SD formulations comprising pluronic F127 (CPG40) exhibited highest solubility in both water and buffer. CPG40 containing drug:polymer in 1:4 ratio fusion (melt) exhibited solubility of $6.57 \pm 0.04 \mathrm{mg} / \mathrm{mL}$ in buffer, which is 45 folds higher than pure drug solubility $(0.146 \pm 0.01 \mathrm{mg} / \mathrm{mL})$. Higher concentrations of polymer lead to increase in drug solubility (Figs 2 and 3).

\section{\% Drug Content and \% Practical Yield of CPG SD}

The drug content and practical yield of all formulations are dependent on angle of repose, which represents uniformity in flow nature of powder blend that, in turn, ensures uniform drug distribution. The \% drug content values of CPG1 to CPG40 are in the range of $94.23 \pm 0.83$ to $99.29 \pm 0.34 \%$. The formulations CPG40 exhibited maximum value of $99.29 \pm 0.34 \%$.

The $\%$ practical yield values of CPG1 to CPG40 are in the range of $97.89 \pm 0.045$ to $98.53 \pm 0.046$. The formulations CPG40 exhibited maximum value of $98.53 \pm 0.046 \%$ (Tables 5 and 6).

\section{In vitro Dissolution Studies}

The dissolution profile of SD CPG40 prepared by fusion (melt) method using 1:4 ratio of drug:polymer (CPG:pluronic F127), showed maximum drug release of 99.14\% in 60 minutes, and found to be the best-optimized formulation, when compared that with other formulations

Table 5: \% drug content and \% practical yield of CPG SD (CPG1-CPG20)

\begin{tabular}{lll}
\hline F No. & Drug content (\%) & \% practical yield \\
\hline CPG1 & $95.23 \pm 0.63$ & $93.45 \pm 0.031$ \\
CPG2 & $97.04 \pm 0.06$ & $97.89 \pm 0.045$ \\
CPG3 & $95.56 \pm 0.14$ & $91.21 \pm 0.018$ \\
CPG4 & $98.11 \pm 1.01$ & $95.67 \pm 0.033$ \\
CPG5 & $94.23 \pm 0.83$ & $94.78 \pm 0.012$ \\
CPG6 & $95.45 \pm 0.31$ & $97.35 \pm 0.056$ \\
CPG7 & $94.78 \pm 0.49$ & $95.15 \pm 0.027$ \\
CPG8 & $97.23 \pm 0.51$ & $92.32 \pm 0.067$ \\
CPG9 & $96.13 \pm 0.56$ & $98.56 \pm 0.017$ \\
CPG10 & $95.23 \pm 0.24$ & $94.25 \pm 0.043$ \\
CPG11 & $97.97 \pm 0.21$ & $98.54 \pm 0.013$ \\
CPG12 & $95.45 \pm 0.76$ & $95.15 \pm 0.019$ \\
CPG13 & $97.45 \pm 0.48$ & $96.56 \pm 0.028$ \\
CPG14 & $96.98 \pm 0.23$ & $93.25 \pm 0.037$ \\
CPG15 & $98.45 \pm 0.36$ & $95.59 \pm 0.026$ \\
CPG16 & $96.45 \pm 0.69$ & $94.45 \pm 0.057$ \\
CPG17 & $95.34 \pm 0.35$ & $93.43 \pm 0.078$ \\
CPG18 & $97.56 \pm 0.23$ & $98.23 \pm 0.046$ \\
CPG19 & $96.29 \pm 0.34$ & $95.61 \pm 0.089$ \\
CPG20 & $97.18 \pm 0.81$ & $97.34 \pm 0.026$ \\
\hline ( $1507 n$ & &
\end{tabular}

${ }^{*}( \pm \mathrm{SD}) \mathrm{n}=3$ and pure drug (32.76\%). Increased dissolution rates of SDs is attributed to more polymer concentration used for

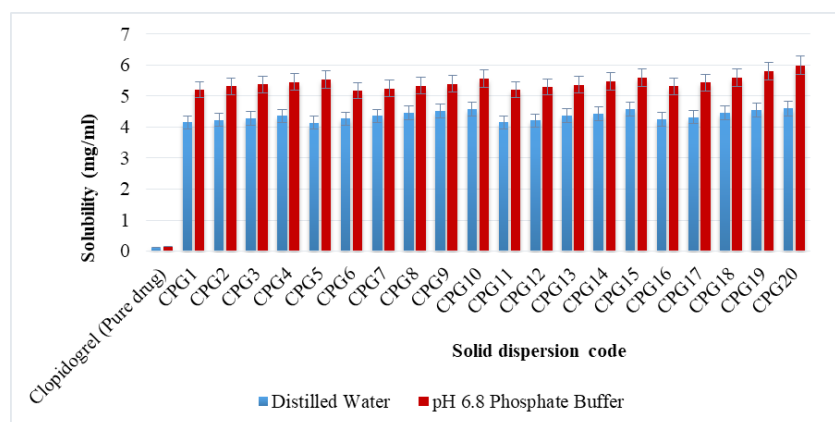

Fig. 2: Solubility studies of CPG SD (CPG1-CPG21); ${ }^{*}( \pm \mathrm{SD}) \mathrm{n}=3$

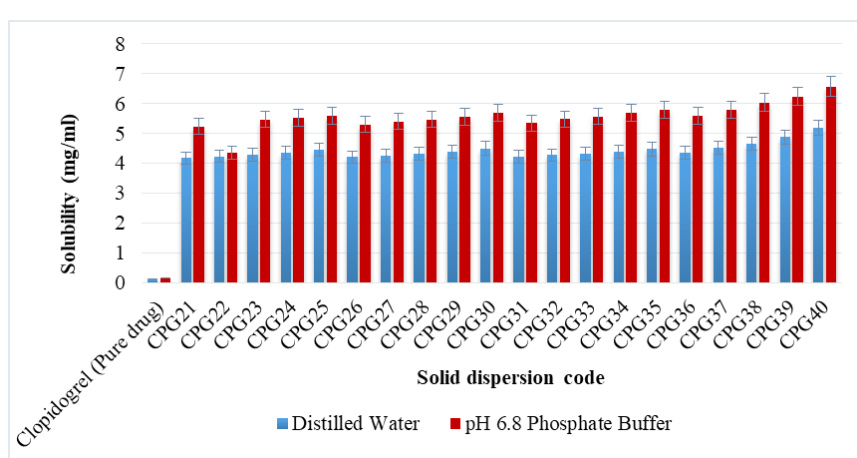

Fig. 3: Solubility studies of CPG SD (CPG21-CPG40); ${ }^{*}( \pm \mathrm{SD}) \mathrm{n}=3$

Table 6: \% drug content and \% practical yield CPG SD (CPG21-CPG40)

\begin{tabular}{lll}
\hline F No. & Drug content $(\%)$ & \% practical yield \\
\hline CPG21 & $97.55 \pm 0.72$ & $98.15 \pm 0.026$ \\
CPG22 & $98.23 \pm 0.18$ & $96.82 \pm 0.059$ \\
CPG23 & $97.23 \pm 0.45$ & $95.81 \pm 0.017$ \\
CPG24 & $97.29 \pm 1.17$ & $97.57 \pm 0.045$ \\
CPG25 & $98.78 \pm 0.31$ & $96.8 \pm 0.037$ \\
CPG26 & $95.96 \pm 0.48$ & $95.53 \pm 0.017$ \\
CPG27 & $96.18 \pm 0.88$ & $94.26 \pm 0.053$ \\
CPG28 & $98.4 \pm 0.22$ & $98.18 \pm 0.042$ \\
CPG29 & $97.27 \pm 0.12$ & $96.56 \pm 0.075$ \\
CPG30 & $96.33 \pm 0.58$ & $95.78 \pm 0.061$ \\
CPG31 & $98.17 \pm 0.28$ & $98.14 \pm 0.026$ \\
CPG32 & $97.62 \pm 0.76$ & $96.44 \pm 0.027$ \\
CPG33 & $98.45 \pm 0.5$ & $97.61 \pm 0.039$ \\
CPG34 & $97.18 \pm 0.68$ & $96.25 \pm 0.059$ \\
CPG35 & $98.45 \pm 0.36$ & $95.63 \pm 0.018$ \\
CPG36 & $97.12 \pm 0.48$ & $96.17 \pm 0.092$ \\
CPG37 & $96.57 \pm 0.39$ & $94.21 \pm 0.067$ \\
CPG38 & $98.34 \pm 0.61$ & $96.79 \pm 0.012$ \\
CPG39 & $98.89 \pm 0.56$ & $98.03 \pm 0.063$ \\
CPG40 & $99.29 \pm 0.34$ & $98.53 \pm 0.046$ \\
\hline ( 5 SD) & &
\end{tabular}

${ }^{*}( \pm \mathrm{SD}) \mathrm{n}=3$ 


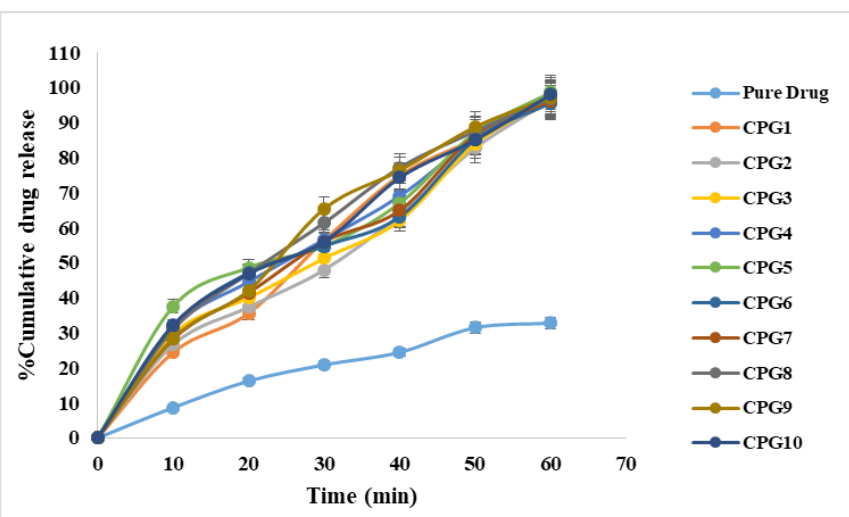

Fig. 4: Dissolution profile of CPG pure drug and CPD SD (CPG1-CPG10); ${ }^{*}( \pm \mathrm{SD}) \mathrm{n}=3$

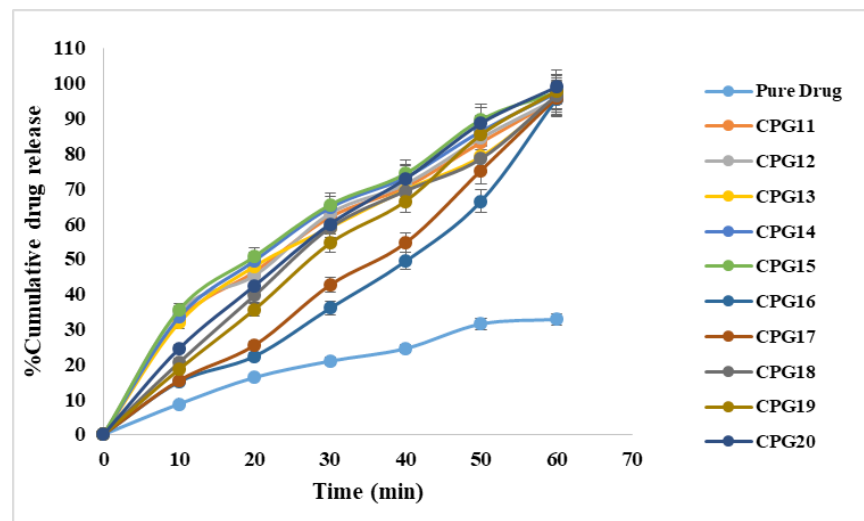

Fig. 5: Dissolution profile of CPG pure drug and CPD SD (CPG11-CPG20); ${ }^{*}( \pm \mathrm{SD}) \mathrm{n}=3$

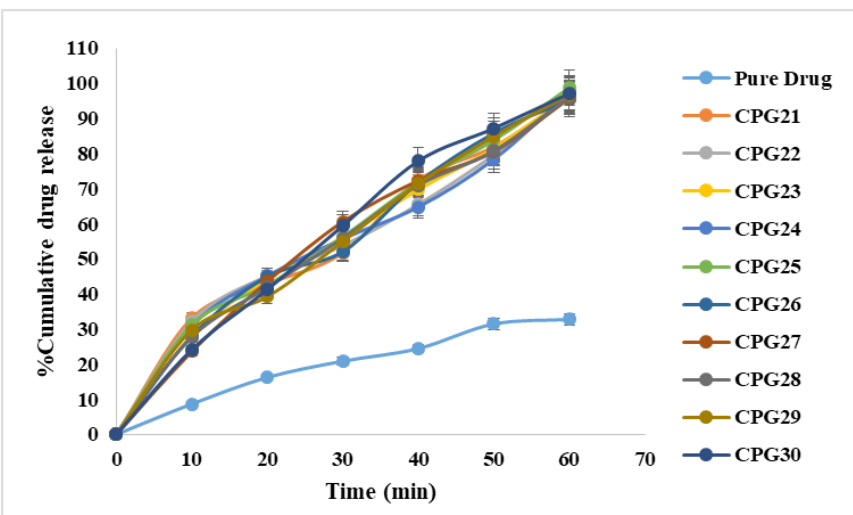

Fig. 6: Dissolution profile of CPG pure drug and CPD SD (CPG21-CPG30); ${ }^{*}( \pm \mathrm{SD}) \mathrm{n}=3$

formulating SDs; it is clearly observed that as the polymer carrier concentration was increased in the formulation the drug release increased accordingly (Figs 4 to 7 ).

\section{FTIR Studies}

The FTIR pure CPG (Fig. 8) showed a peak at $1,753 \mathrm{~cm}^{-1}$ ( $\mathrm{C}=\mathrm{O}$ stretching), $3,012 \mathrm{~cm}^{-1}$ (O-H stretching of the hydrogen sulfate), $3,414 \mathrm{~cm}^{-1}$, and $2,343 \mathrm{~cm}^{-1}$ (N-H stretching), 1,066 , 1,176 , and $1,220 \mathrm{~cm}^{-1}$ (C-O stretching). The FTIR spectra of CPG optimized formulation CPG40 (Fig. 9) showed similar prominent peaks pure drug, and these results indicate the

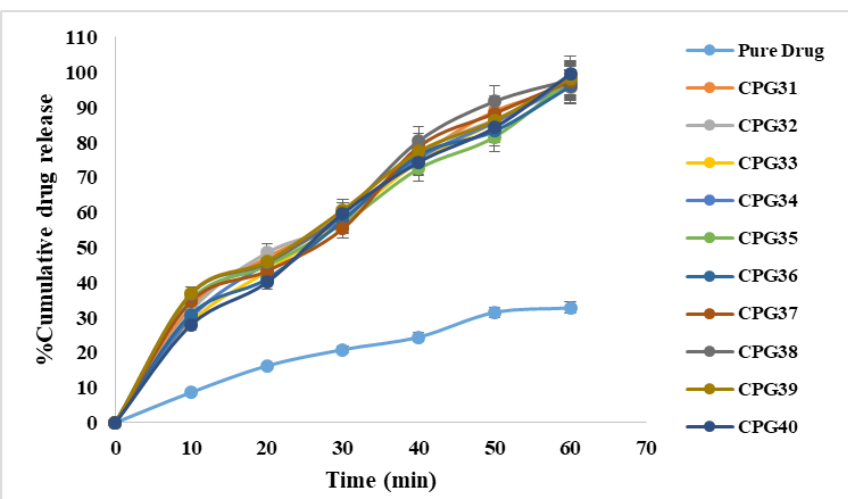

Fig. 7: Dissolution profile of CPG pure drug and CPD SD (CPG31-CPG40); ${ }^{*}( \pm \mathrm{SD}) \mathrm{n}=3$

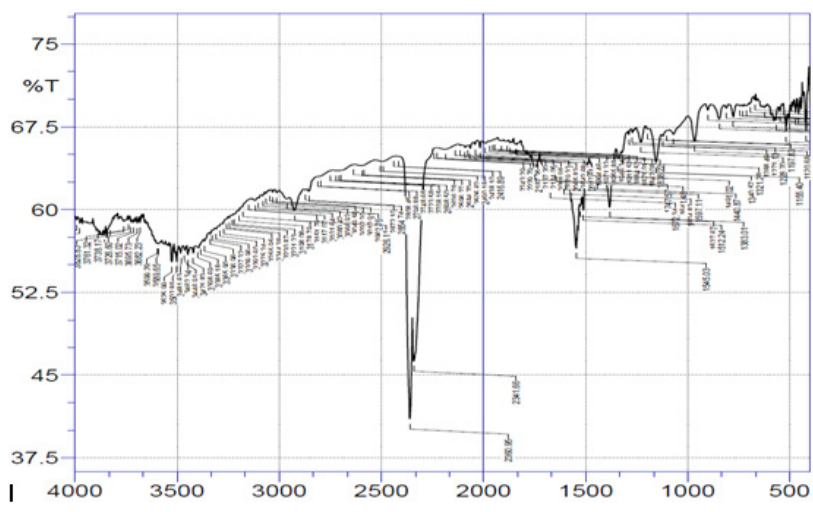

Fig. 8: FTIR of CPG pure drug

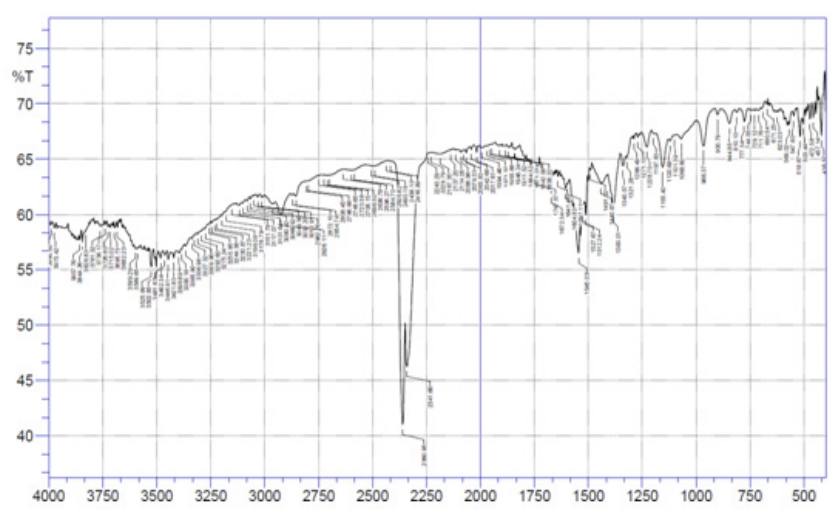

Fig. 9: FTIR spectrum of optimized CPD SD (CPG40)

lack of any chemical interactions among CPG and used excipients in the formulation.

\section{X-Ray Powder Diffraction (XRD)}

The CPG SDs XRD was carried out to find out the crystalline or amorphous states of the drug. The presence of distinct peaks in the spectrum of CPG confirms its crystalline form (Fig. 10A). A change in the diffraction patterns was seen. The pure drug showed sharp peaks, whereas the co-crystals did not show sharp peaks, which suggests that there is interaction. Inter-arrangement of molecules is 


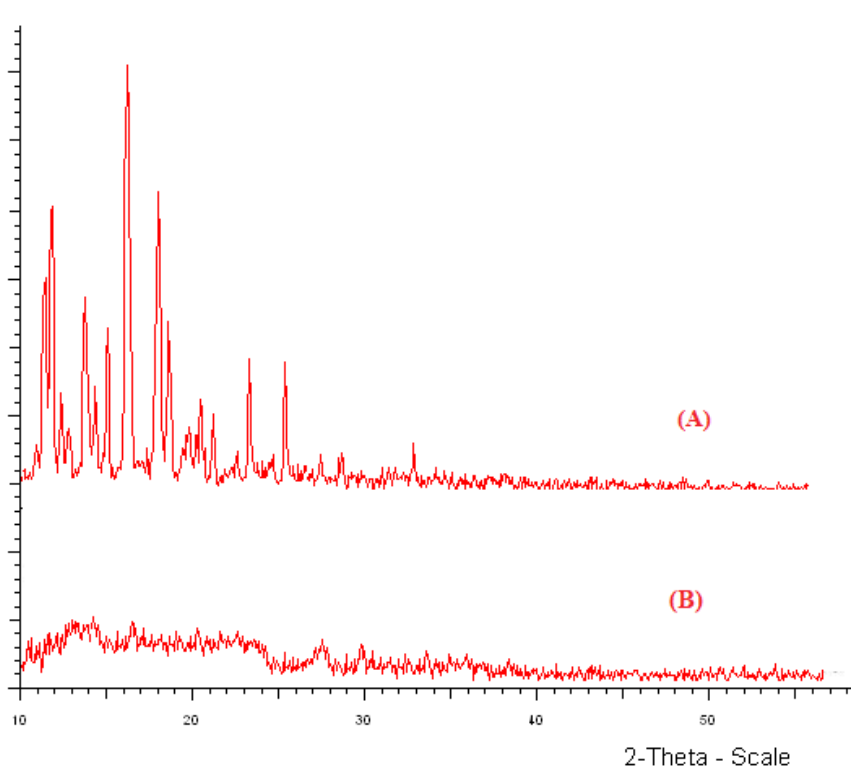

Fig. 10: XRD of CPG pure drug (A); optimized formulation CPG40 (B)

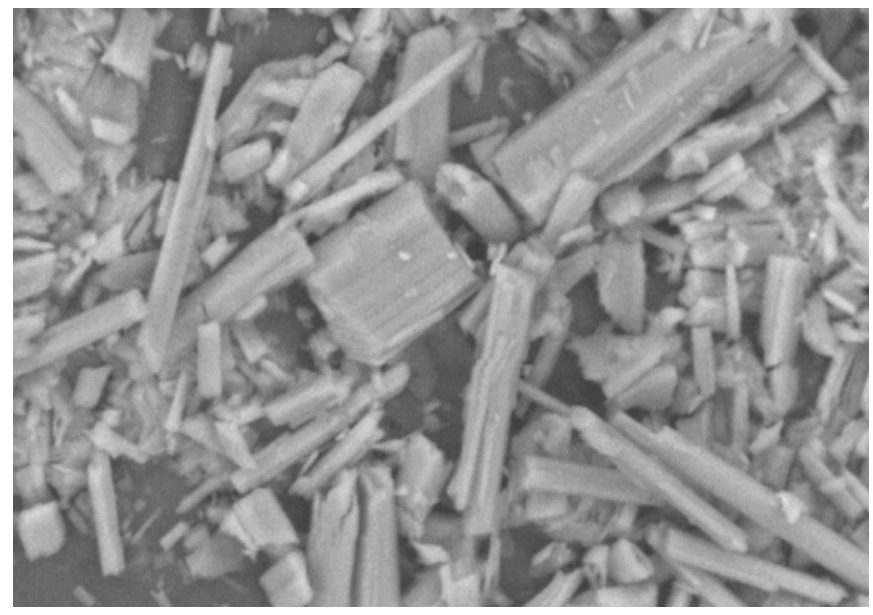

Fig. 11: SEM image of pure CPG drug

indicated by different peak locations of the co-crystals with respect to the pure drug, hence, it proves the formation of a new phase. The augmentation in dissolution rate of drug from CPG40 is due to a decline in the crystallinity of the drug (Fig. 10B).

\section{SEM Studies}

The SEM data indicates smooth surface and irregular shape of drug crystals (Fig. 11). The surface of drug in SD formulation is porous and appeared a mixed mass. The drug particles seem to be completely incorporated into the formulation with the dispersion looking like a matrix. The results ensure the complete dispersion of the drug (Fig. 12).

\section{Stability Studies}

Optimized formulation CPG40 was loaded for stability studies at $40 \pm 2{ }^{\circ} \mathrm{C} / 75 \pm 5 \% \mathrm{RH}$, and found stable. There was no noteworthy variation in \% drug content, and in vitro drug release was observed (Table 7).

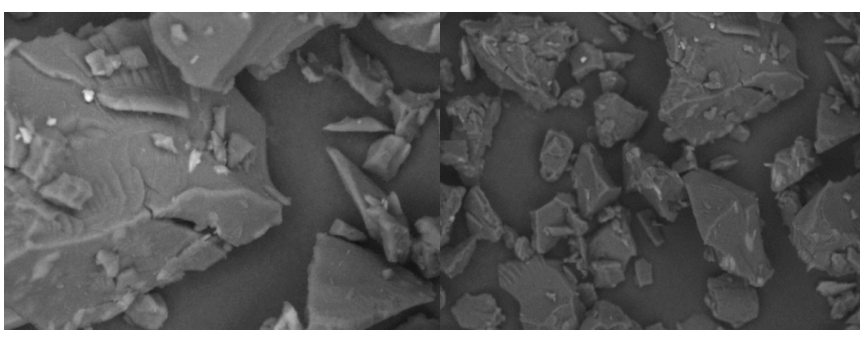

Fig. 12: SEM image of optimized CPG40

Table 7: Stability study of optimized CPG SD (CPG40)

\begin{tabular}{lll}
\hline Time & \% drug content & In vitro drug release (\%) \\
\hline 0 & $99.29 \pm 0.34$ & $99.14 \pm 3.87$ \\
30 & $98.86 \pm 0.53$ & $99.09 \pm 0.67$ \\
60 & $97.92 \pm 0.47$ & $98.89 \pm 0.42$ \\
90 & $97.47 \pm 0.97$ & $98.21 \pm 0.87$ \\
${ }^{*}( \pm \mathrm{SD}) \mathrm{n}=3$ & &
\end{tabular}

\section{Discussion}

The CPG SDs prepared by solvent evaporation and fusion melt method. A total of 40 formulations were prepared using different hydrophilic carrier polymers, and all the formulations exhibited better drug dissolution in comparison to pure drug. The solubility of CPG was found to be highest in polymer pluronic F127 in phosphate buffer $\mathrm{pH}$ 6.8, which was almost 26 folds and the same reflected in the formulation CPG40, which showed the highest release of $99.54 \%$ in 60 minutes containing 1:4 ratio of CPG:pluronic F127 prepared by fusion melt method and found to be the best-optimized formulation. Solubility of CPG was also increased by 45 folds in SD formulation CPG40 when compared to pure drug. XRD and SEM studies manifest that CPG40 is in amorphous form that achieved better dissolution, when compared to the pure drug. Therefore, from the results obtained after a thorough investigation, it was assessed that SD prepared by fusion melt method showed better drug release than the solvent evaporation method and resulted in enrichment of solubility, thereby, improvement in drug release of sparingly water-soluble drug CPG.

\section{REFERENCES}

1. Vasconcelos T, Sarmento B, Costa P. Solid dispersions as strategy to improve oral bioavailability of poor water soluble drugs. Drug Dis Today. 2007;12(23-24):1068-1075.

2. Perioli L, D'Alba G, Pagano C. New oral solid dosage form for furosemide oral administration. Eur J Pharm Biopharm. 2012;80(3):621-629.

3. Ali AA, Gorashi AS. Absorption and dissolution of nitrofurantoin from different experimental formulations. Int J Pharm. 1984;19: 297-306.

4. Ahire BR, Rane BR, Bakliwal SR, Pawar SP. Solubility enhancement of poorly water soluble drug by solid dispersion techniques. Int J Pharm Tech Res. 2010;2:2007-2015.

5. Bang LM, Chapman TM, Goa KL. Clopidogrel: A review of its efficacy in the management of hypertension. Drugs. 2003;63:2449-2472.

6. Borghi C. Clopidogrel in hypertension. Vasular Health and Risk Management. 2005;1(3):173-182. 
7. Patel J, Kevin G, Anjali P, Mihir R, Navin S. Design and development of a self-nanoemulsifying drug delivery system for telmisartan for oral drug delivery. Int J Pharm Investig. 2011;1(2):112-118.

8. Arora SC, Sharma PK, Irchhaiya R, Khatkar A, Singh N, Gagoria J. Development, characterization and solubility study of solid dispersions of Cefuroxime Axetil by the solvent evaporation method. J Adv Pharm Technol Res. 2010;1(3):326-329.

9. Anant P, Manish M, Amit KT, Bhaskar C, Kadam SS. Preparation and characterization of flurbiprofen beads by melt solidification technique. AAPS Pharm Sci Tech. 2003;4(4):65.

10. Saravanan M, Natraj KS, Ganesh KS. The effect of tablet formulation and hardness on in vitro release of cephalexin from Eudragit L100 based extended release tablets. Biol Pharm Bull. 2002;25:4541-4545.

11. Afrasim M, Shivakua HG. Formulation of Sustained-release diltiazem matrix tablets using hydrophilic gum blends. Trop J Pharm Res. 2010;9:283-291.

12. Mohammadi H, Hemanath KV. Formulation and evaluation of solid dispersion incorporated fast disintegrating tablets of tenoxicam using design of experiment. Int J Pharm Sci Drug Res. 2019;11(1):35-44.

13. Vanshiv SD, Rao MRP, Sonar GS. Physicochemical characterization and in vitro dissolution of Domperidone by solid dispersion technique. Ind J Pharm Edu Res. 2009;43(1):86-90.

14. Subhash K, Bidkar SJ, Dama GY. Formulation and evaluation of ciprofloxacin solid dispersion controlled release floating capsules for solubility improvement. Indian J Pharm Biol Res. 2017;5(3):7-16.

15. Batra V, Shirolkar VS, Mahaparale PR. Solubility and dissolution enhancement of glipizide by solid dispersion technique. Ind J Pharm Edu Res. 2008;42(4):373-378.

16. Yadav B, Tanwar YS. Development, characterization and in vitro evaluation of flurbiprofen solid dispersions using polyethylene glycols as carrier. J App Pharm Sci. 2016;6(04):60-66.

17. Mohammadi G, Barzegar-Jalali A, Khosro A. Development and characterization of solid dispersion for dissolution improvement of furosemide by co grinding method. Adv Pharm Bull. 2014;4(4):391-399.

18. Soliman MS, Khan MA. Preparation and in vitro characterization of a semi-solid dispersion of flurbiprofen with Gelucire44/14 and Labrasol. Pharmazie. 2005;60(4):288-293.

19. Shailendra KS, SoukaryaSom, Shankhwar U. Formulation and optimization of solid dispersion of Clopidogrel with PEG 6000 J Appl Pharm Sci. 2011;1(8):217-226.

20. Shaikh FI, Patel VB. Enhancement of dissolution of clopidogrel Hydrochloride using solid dispersion technique. Res J Recent Sci. 2015;4:299-307.

21. Valizadeh H, Nokhodchi A, Qarakhani N, Zakeri-Milani P, Azarmi S, Hassanzadeh D, et al. Physicochemical characterization of solid dispersions of indomethacin with PEG 6000, Myrj 52, lactose, sorbitol, dextrin, and Eudragit E100. Drug Dev Ind Pharm. 2004;30(3):303-317.

HOW TO CITE THIS ARTICLE: Khader MA, Salfi R. Enhancement of solubility and dissolution profile of clopidogrel by various solid dispersion formulations. Int. J. Pharm. Sci. Drug Res. 2020;12(4):377-383. DOI: 10.25004/IJPSDR.2020.120410 